\title{
Striatonigral Degeneration: Iron Deposition in Putamen Correlates with the Slit-like Void Signal of Magnetic Resonance Imaging
}

\author{
Anthony E. Lang, Terry Curran, John Provias and Catherine Bergeron
}

\begin{abstract}
We report three patients with striatonigral degeneration highlighting the correlation between magnetic resonance imaging (MRI) and the pathological changes. The "slit-like void signal" observed in the putamen is typical of striatonigral degeneration and can be used to assist diagnosis during life. Our histochemical studies support the concept that increased iron deposition in the putamen is responsible for this MRI picture.
\end{abstract}

Résumé: Dégénérescence striatonigrale: les dépôts de fer dans le putamen sont corrélés au signal ("slit-like void") de l'imagerie par résonance magnétique. Nous rapportons les cas de trois patients atteints de dégénérescence striatonigrale qui illustrent la corrélation qui existe entre l'imagerie par résonance magnétique nucléaire (RMN) et les changements anatomopathologiques. Le signal ("slit-like void") observé dans le putamen est typique de la dégénérescence striatonigrale et peut être utilisé pour aider au diagnostic du vivant du patient. Nos études histochimiques appuient le concept que des dépôts accrus de fer dans le putamen sont responsables de cette image à la RMN.

Can. J. Neurol. Sci. 1994; $21: 311-318$

Diseases included under the term "multiple system atrophy" (MSA), in particular striatonigral degeneration (SND), are notoriously difficult to differentiate clinically from idiopathic Parkinson's disease (PD). Often they are not correctly diagnosed until necropsy, even when stringent antemortem inclusion and exclusion criteria are applied.' Recently, abnormalities have been noted with magnetic resonance imaging (MRl) which may assist in differentiating "Parkinson-plus" syndromes from PD. ${ }^{2-5}$ The most extensively documented MRI abnormality has been the presence of an accentuated low signal intensity, presumed to be related to iron deposition, in the putamen. However, the significance of these abnormalities and their structural basis is uncertain. We present three patients with SND where the MRI appearance is correlated with the pathological changes, in particular iron deposition.

\section{Case Studies}

\section{Patient 1}

This right-handed female presented at the age of 65 with a one year history of tremor, clumsiness and abnormal posturing in the left arm. Neurological examination revealed a fine facial tremor and action tremor of the left arm, generalized rigidity and mild bradykinesia. Shortly thereafter she began to experience occasional spontaneous falls. A diagnosis of Parkinson's disease was made and levodopa was started with an initial definite improvement but the benefit waned over the next six months. There was no further improvement with the addition of bromocriptine. Her disability continued to progress with generalized bradykinesia, rigidity, falls and later dystonic posturing in the left hemibody which may have been in part aggravated by levodopa, especially the facial involvement. The diagnosis of MSA, likely SND. was entertained. MRI using a 1.5 Tesla GE unit demonstrated signal attenuation in the putamen on T1 imaging (Figure $1 \mathrm{~A}$ ) and marked signal loss and atrophy of the putamen on the T2 scan (Figure lB). The patient died six years after the onset of her symptoms from respiratory complications of a severe bedbound parkinsonian state.

\section{Patient 2}

This right-handed male presented at the age of 64 with speech disturbance and loss of dexterity in both hands. The neurological examination revealed drooling, generalized bradykinesia and micrographia. The diagnosis of PD was considered and the patient was started on levodopa. His response to levodopa, amantadine and trihexyphenidyl was poor and his symptoms continued to progress. He was first seen at our hospital at the age of 68 and the examination revealed severe generalized bradykinesia and rigidity, prominent dysarthria, mild actioninduced facial myoclonus, stimulus sensitive and action-induced limb myoclonus and a left Horner's syndrome. There were no complaints or features of dysautonomia. The diagnosis of SND was considered and the MRI demonstrated abnormalities in the region of the putamen similar to those seen in patient 1 (Figure 2A \& B). Positron emission tomography with $6\left[{ }^{18} \mathrm{~F}\right]$ fluoro-l-dopa (F-dopa) and $\left[{ }^{18} \mathrm{~F}\right]$ fluorodeoxyglucose

From the Morton and Gloria Shulman Movement Disorder Centre (A.E.L., T.C.); Division of Neurology (A.E.L., T.C.) and Department of Pathology (Neuropathology) (J.P., C.B.) The Toronto Hospital, Toronto.

RECEIVED MARCH 28. 1994. ACCEPTED IN FINAL FORM JUNE 17, 1994.

Reprint requests 10: Anthony E. Lang, M.D., Division of Neurology, The Toronto Hospital, 399 Bathurst Street. MP 11-304, Toronto, Ontario. Canada MST 2S8 
(FDG) were performed (courtesy of Dr. E.S. Garnett) using techniques previously described.$^{67}$ F-dopa scan demonstrated markedly reduced accumulation of isotope in both putamen while uptake in the caudate was preserved, changes similar to those seen in typical PD. However, FDG scan demonstrated bilateral absence of glucose accumulation in the putamen. The patient died seven years after the onset of his symptoms at the age of 71 .

\section{Patient 3}

This right-handed woman presented at the age of 41 with a $I$ year history of generalized slowing, clumsiness in the hands interfering with

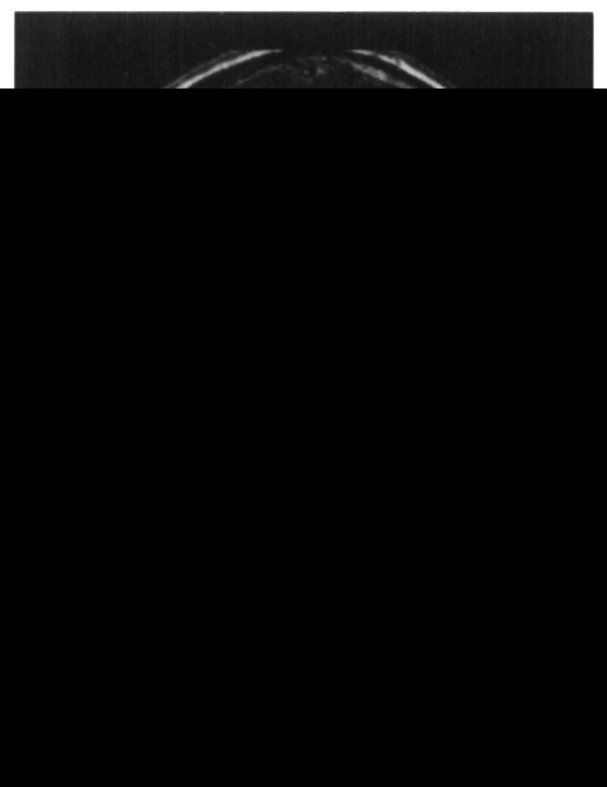

Figure 1A: Coronal TI scan in patient \#I demonstrating signal attenuation in the right putamen. The changes in the left putamen are not as well seen due to rotation of the head in the scanner.

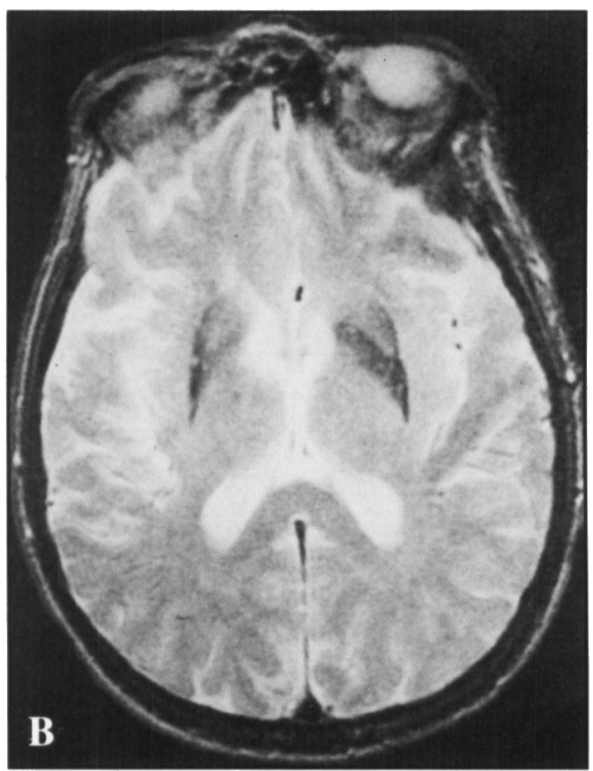

Figure 1B: Axial T2 scan showing a "slit-like void signal" in the region of the putamen bilaterally due to marked signal loss and atrophy. There is a suggestion of a border of hyperintense signal, particularly on the left. activities of daily living and slowness and unsteadiness of gait. Examination at that time revealed hypophonia, a masked face, moderate rigidity in the neck and limbs, moderate to marked bradykinesia, worse on the right side, a slight tremor in the lower face and tongue and a moderate postural and action tremor in the upper limbs but no typical resting tremor. She was unsteady on tandem gait but this was thought to be primarily related to poor postural stability and she had a combination of retropulsion and falling on posterior displacement. Amantadine initially resulted in a notable improvement in her rigidity but not tremor. Levodopa improved both symptoms and soon after beginning the drug she experienced wearing off with increased symptoms occurring 3 to

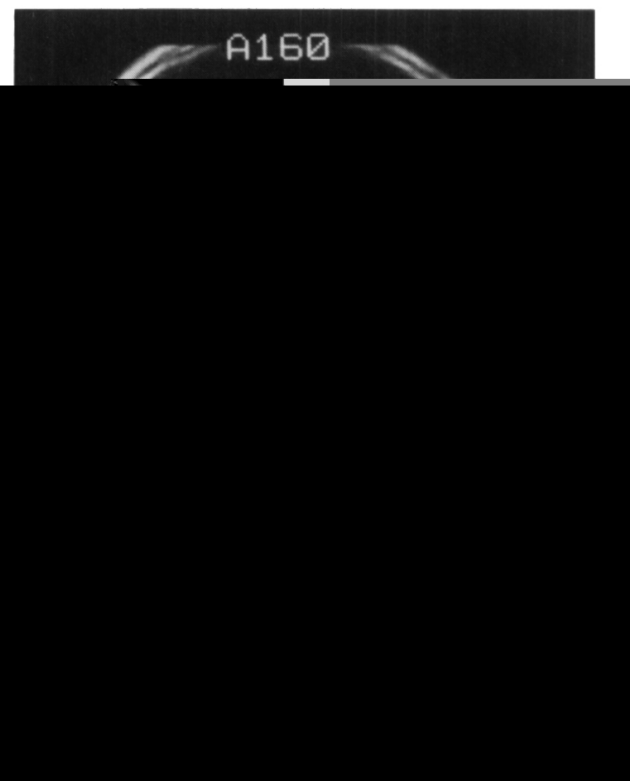

Figure 2A: Axial TI scan in patient \#2 showing attenuation of signal in the putamen bilaterally (arrows), most prominent in the dorsal regions.

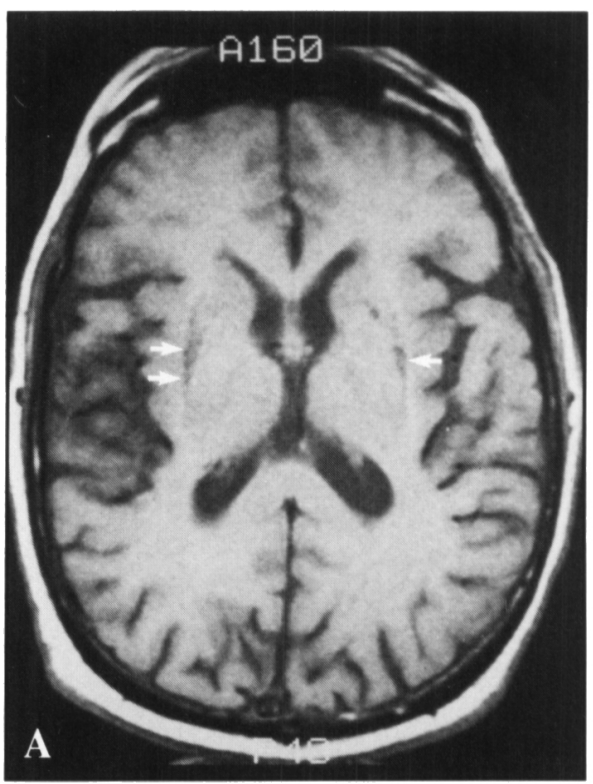

Figure 2B: Axial T2 scan in patient \#2 showing marked loss of signal (especially on the left) and atrophy of the putamen bilaterally. 
$31 / 2$ hours after each dosage. Bromocriptine was poorly tolerated. The dosage of L-dopa was gradually increased because of progressive difficulty with self care activities and ambulation. She also developed frequent freezing of gait and occasional morning foot dystonia. Two years after the onset of her symptoms, 8 months after beginning levodopa, she developed episodes of facial dystonia and dyskinesias in her limbs, particularly on the right side. At times the dyskinesia seemed to have a diphasic pattern in response to her medication. The action-induced cranial dystonia gradually worsened over the next few months. While taking 1 gram of levodopa/carbidopa per day any attempt to speak resulted in severe tonic contraction of the obicularis oculi associated with mouth opening and severe grimacing and distortion of all facial muscles. She became completely mute and unable to produce any sound at this time. Any distraction such as calling her name or asking her to point to the examiner's finger immediately resolved the facial dystonia; however, any attempt to speak caused the dystonia to return just as quickly. These symptoms improved markedly as the dosage of levodopa was reduced.

All symptoms continued to progress and by 4 years after the onset of her symptoms she was chair bound, completely anarthric and required full care. She developed a tonic extension posture of the neck and required fitting with a brace to assist in feeding. Extraocular movements remained full. She developed pronounced "apraxia" of lid opening and closure. At no time did she seem to suffer from cognitive decline or psychiatric side effects from her medication. She developed stimulus-sensitive myoclonus of the hands first noted within a year of her presentation and in the last year of life this was elicitable both in the hands and in the feet. Two years after the onset of her motor symptoms she complained of urinary frequency and occasional episodes of urinary incontinence. However, bowel and bladder difficulties were never prominent and she had no features of orthostatic hypotension. She died due to the complications of a severe debilitated bedbound state 6 years after the onset of her symptoms. MRI performed $21 / 2$ years after the onset of her symptoms demonstrated abnormalities in the region of the putamen similar to those seen in patients 1 and 2 (Figure 3A \& B).

\section{NEUROPATHOLOGY}

\section{Methods}

In patients 1 and 3, one hemisphere was fixed in $10 \%$ neutral buffered formalin, while the other was frozen at $-70^{\circ} \mathrm{C}$; in patient 2 the entire brain was formalin fixed. Multiple blocks were taken from cerebral cortex, cerebellum including dentate nucleus, hippocampus and amygdala. The basal ganglia, midbrain, pons, medulla and spinal cord were also sampled at multiple levels and paraffin processed in routine fashion. Sections were cut at 7 microns and stained with hematoxylin and eosin (H\&E), Perls' stain for iron, Perls' with diaminobenzidine (DAB) enhancement for iron, Von Kossa for calcium salts, periodic acid-Schiff (PAS) as well as immunohistochemically with antisera to glial fibrillary protein (GFAP), neuron specific enolase (NSE) and ferritin.

\section{Gross Examination}

The brain weighed 1290 grams fresh in patient \#1, and 1560 grams in \#2 after formalin fixation. In patient \#3, the right half of the brain which was formalin fixed weighed 570 grams. The gross findings were similar in all cases. The external appearance of the brain was unremarkable. Coronal sections of the hemisphere(s) revealed marked atrophy of the putamen which was reduced to a narrow slit-like structure with a diffuse brownish discoloration, most marked posteriorly and laterally (Figure 4). The caudate nucleus was unremarkable. The substantia nigra (SN) showed moderate to severe loss of pigmentation and tissue volume, most marked in the lateral aspects. In addition, patients \#I and \#3 revealed a mild degree of atrophy of the globus pal-
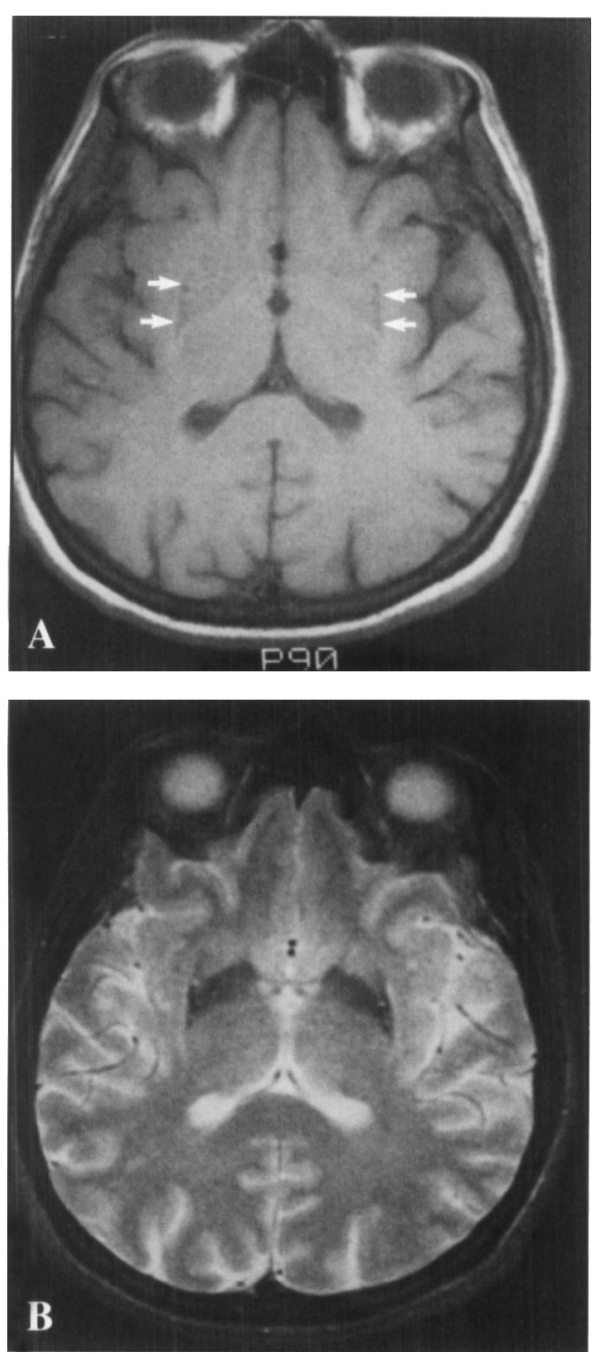

Figure 3A \& B: Axial TI (with arrows outlining the region of signal attenuation) and $T 2 M R I$ scans in patient $\# 3$ demonstrating changes similar to those in patients 1 \& 2 . A lateral border of increased signal on 72 scan is also evident as are punctate areas of increased signal within the region of signal loss (?perivascular spaces vs. regions of cystic degeneration)

lidus, with no change in pigmentation, and patient \#3 showed mild atrophy of the basis pontis.

\section{Histological Examination}

The pathological changes were essentially similar in all cases. The putamen revealed extensive neuronal loss, with severe loss of smaller neurons and occasional large residual neurons (Figure 5). Marked reactive astrocytic gliosis was present, often with a prominent gemistocytic appearance. Although these changes were widespread in the putamen, they were most severe in the dorsolateral and posterior aspects. There was a striking lateral to medial gradient with the more medial putaminal aspects less severely damaged. The caudate nucleus showed only very minimal neuronal loss and astrocytic gliosis. The globus pallidus showed degeneration of putaminopallidal fibres with neuronal loss and reactive astrocytic gliosis. The substantia nigra showed a moderate to severe loss of neurons and neuromelanin pigment, most marked in the lateral aspect of the zona compacta. This was accompanied by astrocytic gliosis and microglial proliferation. 


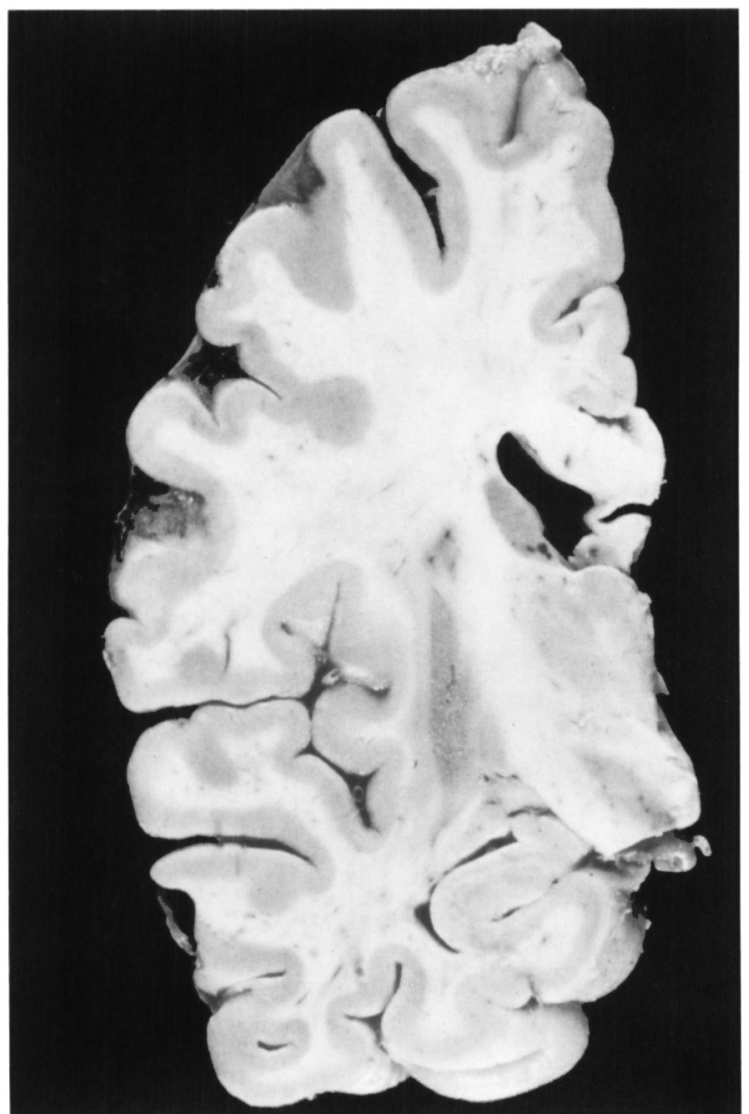

Figure 4: Gross Pathology: Coronal section of cerebral hemisphere with slit-like atrophy and brown pigmentation of the putamen in patient \#.

In patients \#2 and 3, there was additional mild neuronal loss and astrocytic gliosis of the dorsal motor nucleus of the vagus nerve, neurons of the basis pontis, inferior olives, and the locus ceruleus. The cerebellar cortex showed minimal loss of Purkinje cells. The spinal cord showed marked loss of neurons within the intermediolateral cell column as well as the nucleus of Onuf, while motor neurons of the ventral horns were relatively preserved. In patient \#2, a rare Lewy body was observed in locus ceruleus.

In all patients small oligodendroglial eosinophilic cytoplasmic inclusions were present, characterized by marked argyrophilia using the Bielschowsky silver stain (Figure 6). They were widely distributed throughout the brain, particularly in the interfascicular oligodendrocytes of hemispheric white matter tracts.

Extensive brown pigment deposition was present throughout the putamen, with more pigment deposited in the more severely affected dorsolateral and posterior aspects. This pigment which, on $\mathrm{H} / \mathrm{E}$ stain appeared predominantly extracellular, showed intense positive staining with Perls' stain for ferric iron with and without DAB intensification (Figure 7). The pigments rarely stained with PAS-D and were unreactive with Von Kossa stain. The pigment was not seen to any significant extent within the caudate nucleus and showed minimal deposition in the lateral aspects of the substantia nigra zona compacta.

Immunohistochemistry with antisera to GFAP confirmed the presence of marked diffuse reactive astrogliosis, again more

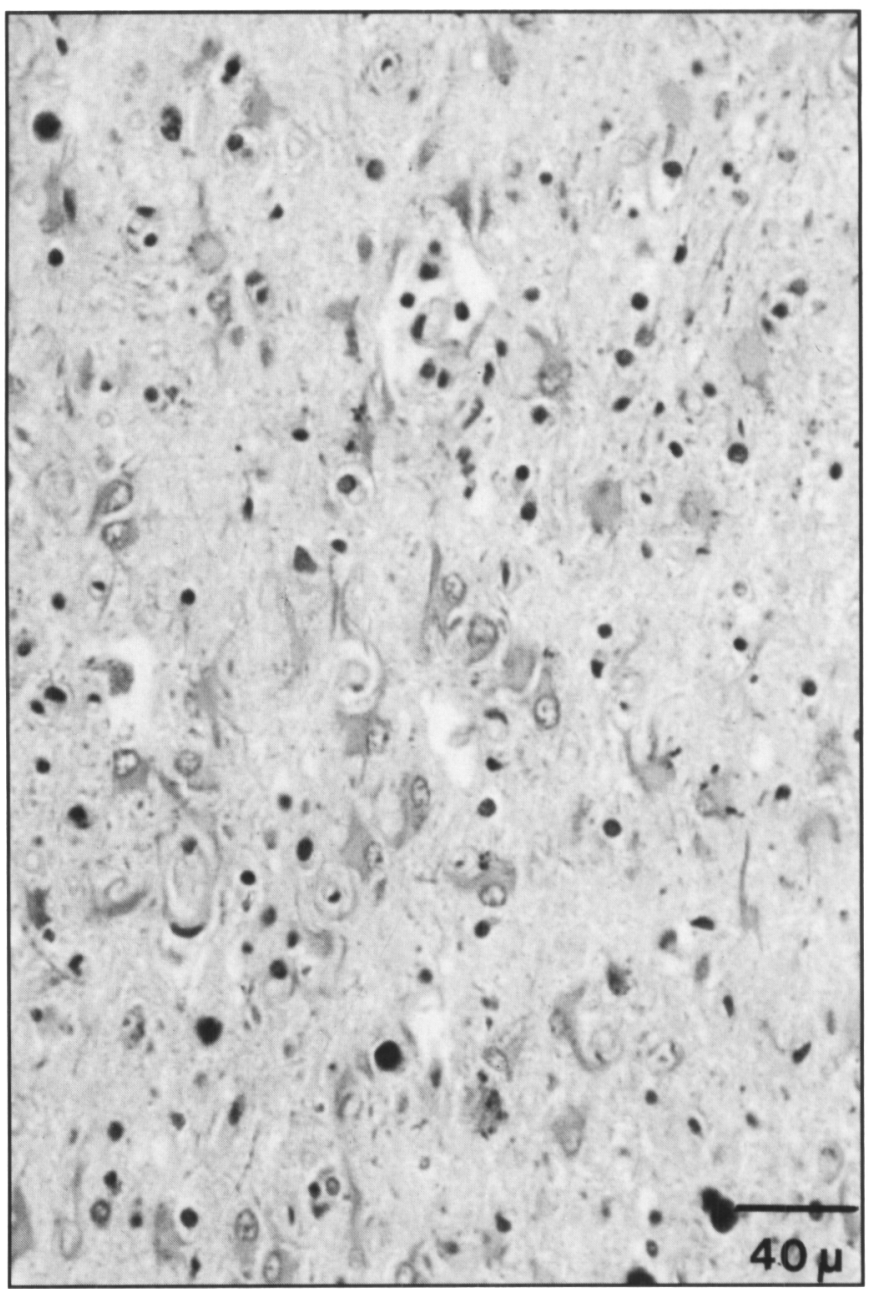

Figure 5: Severe neuronal loss and reactive astrogliosis, $(H \& E, 250 X)$ in dorsolateral zone of the putamen of patient \#2.

severe in the dorsolateral aspects of the putamen. Combined GFAP/Perls' stain showed little or no iron within reactive astrocytes (Figure 8 ). Antisera to ferritin revealed a pronounced diffuse increased staining of microglial cells and associated processes corresponding to the areas of neuronal loss. Double labelling with ferritin and Perls' stain showed much of the iron in the soma and processes of microglial cells (Figure 9). Combined NSE/Perls' showed that the few residual large putaminal neurons very occasionally showed small granular deposits of iron after DAB intensification (Figure 10).

\section{Discussion}

All 3 patients had the typical neuropathological findings of striatonigral degeneration. Patients \#2 and 3 showed additional features of MSA in brainstem and spinal cord. Oligodendroglial cytoplasmic inclusions were present in all cases. These have recently been described in $\mathrm{MSA}^{8}$ and appear to be an important pathological hallmark for this disease. ${ }^{9}$ Their significance, if any, remains unknown although oligodendrocytes may play a role in iron transport and storage within the CNS. ${ }^{8.10,11}$

All 3 patients presented with a parkinsonian syndrome which was either poorly (patient \#2) or transiently (patients \#1 and 3) 


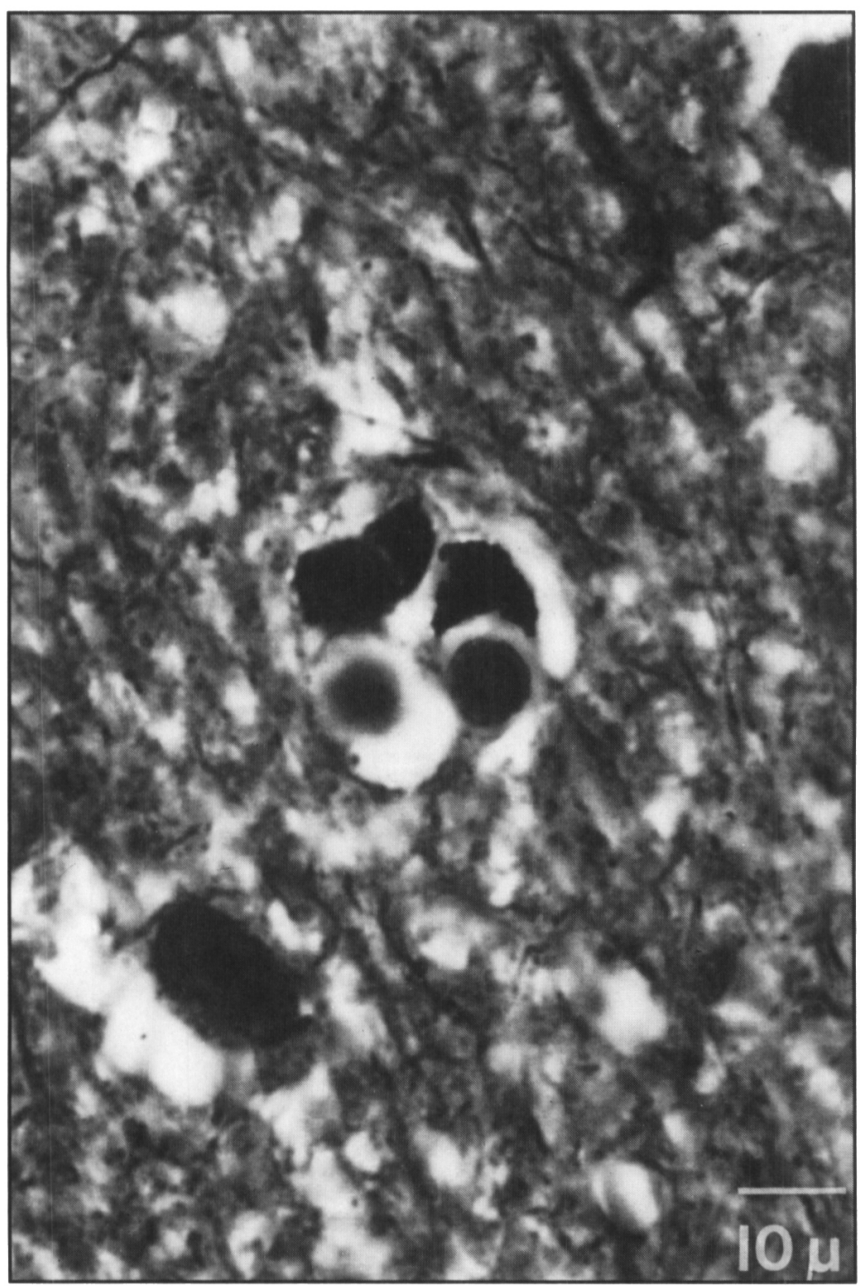

Figure 6: White matter oligodendrocytes with argyrophilic crescentic cytoplasmic inclusions, characteristic of MSA. (Bielschowsky, 1000X). From hemispheric white matter in patient \#3.

responsive to levodopa. In both patients obtaining some benefit from levodopa, prominent (? peak-dose) cranial dystonia complicated this treatment, especially later in the course when response to the drug had greatly diminished. The face may be a particularly common site of levodopa-induced dyskinesias in MSA and when facial dyskinesia/dystonia occurs in association with a modest or negligible motor response, a diagnosis of MSA must be strongly considered. ${ }^{12}$ Stimulus-sensitive myoclonus in the hands was readily elicitable in both patients in whom it was sought (patients \#2 and 3 ). When this feature is present in a parkinsonian patient, especially early in the course of the illness, in the absence of cognitive disturbances, intercurrent illness and other neurological abnormalities (e.g., apraxia or cortical sensory disturbances suggesting cortical-basal ganglionic degeneration), ${ }^{13}$ it may be an important clue to the diagnosis of MSA. ${ }^{14,15}$

These clinical features, however, cannot be taken as definitive evidence for a diagnosis of MSA. Modern imaging may provide further support. Abnormalities of ${ }^{18} \mathrm{~F}$-dopa PET scanning in MSA are very similar to those found in PD. ${ }^{16,17}$ The FDG PET scan in patient \#2 showed severe putaminal hypometabolism. Eidelberg and his colleagues have demonstrated that this finding is typical of SND and it both distinguishes this

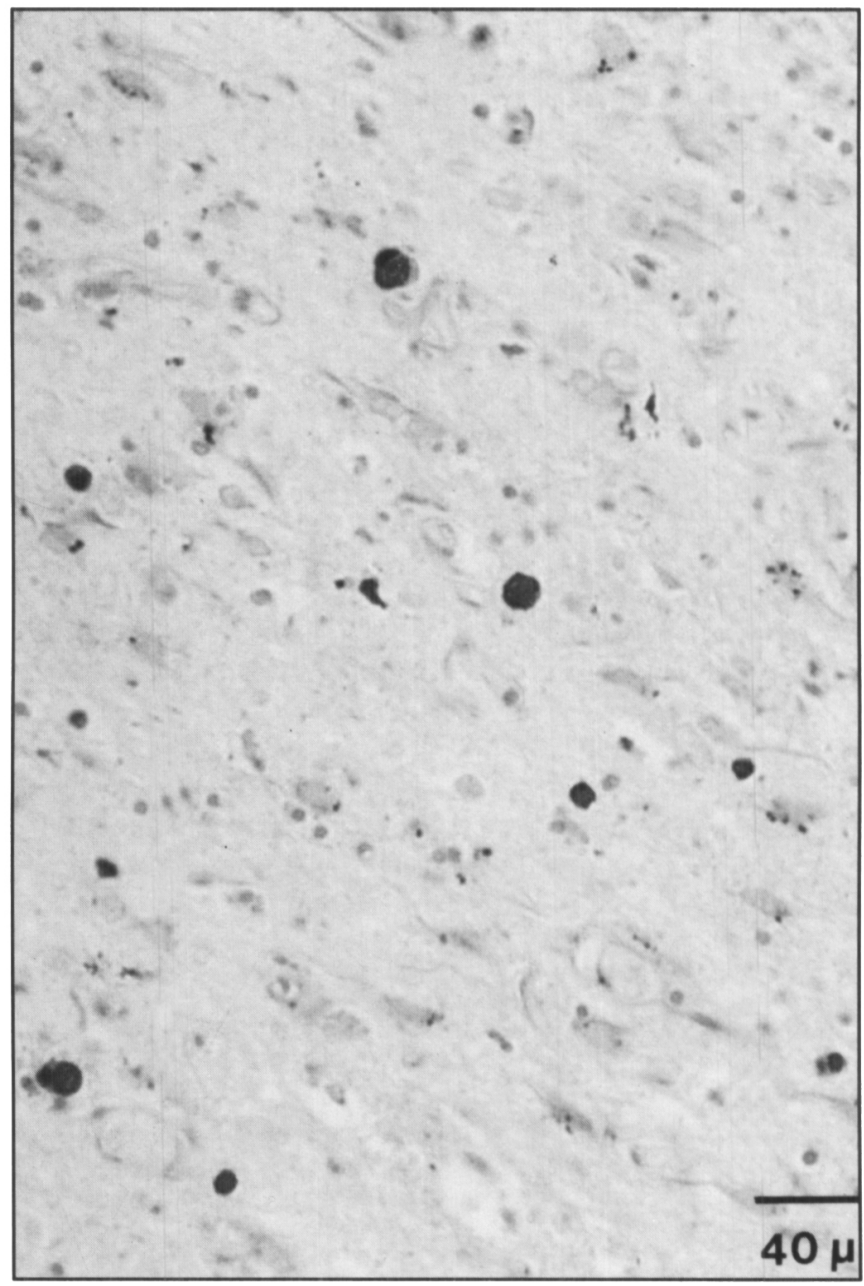

Figure 7: Extensive iron deposition in neuropil of the putamen with concretions. (Perls' stain for iron, 250X). From patient \#2.

disorder from Parkinson's disease, where putaminal metabolism is normal, and correlates with quantitative ratings of the motor disability. ${ }^{18}$ However, PET scanning is not readily available for routine diagnostic purposes while MRI scanning is, in most large centres. An important aspect of our observations is the MRI-pathological correlation which supports the diagnostic utility of MRI for SND. All 3 patients showed significant atrophy and signal loss in the putamen on $\mathrm{T} 1$ images. Heavily weighted T2 images revealed prominent hypointense signals in the putamen producing a "slit-like void signal" which we believe is characteristic if not pathognomonic of this disorder. Given the very short $\mathrm{T} 2$ relaxation in the putamen, it is likely that $\mathrm{T} 2$ effects caused the reduced signal in this region on $\mathrm{T} 1$ weighted images. To date there has been only one other pathologically proven case reported with MRI correlation which also had this T2 "slit-like void signal". 19 This combined experience favours the concept that MRI may be used to support a diagnosis of SND in life. However, more examples will have to be studied to provide an accurate assessment of the specificity and sensitivity of this finding. To date the specificity of the finding for SND seems to be quite high. The sensitivity may be lower since Eidelberg et al. ${ }^{18}$ found similar MRI abnormalities in only 4 of 


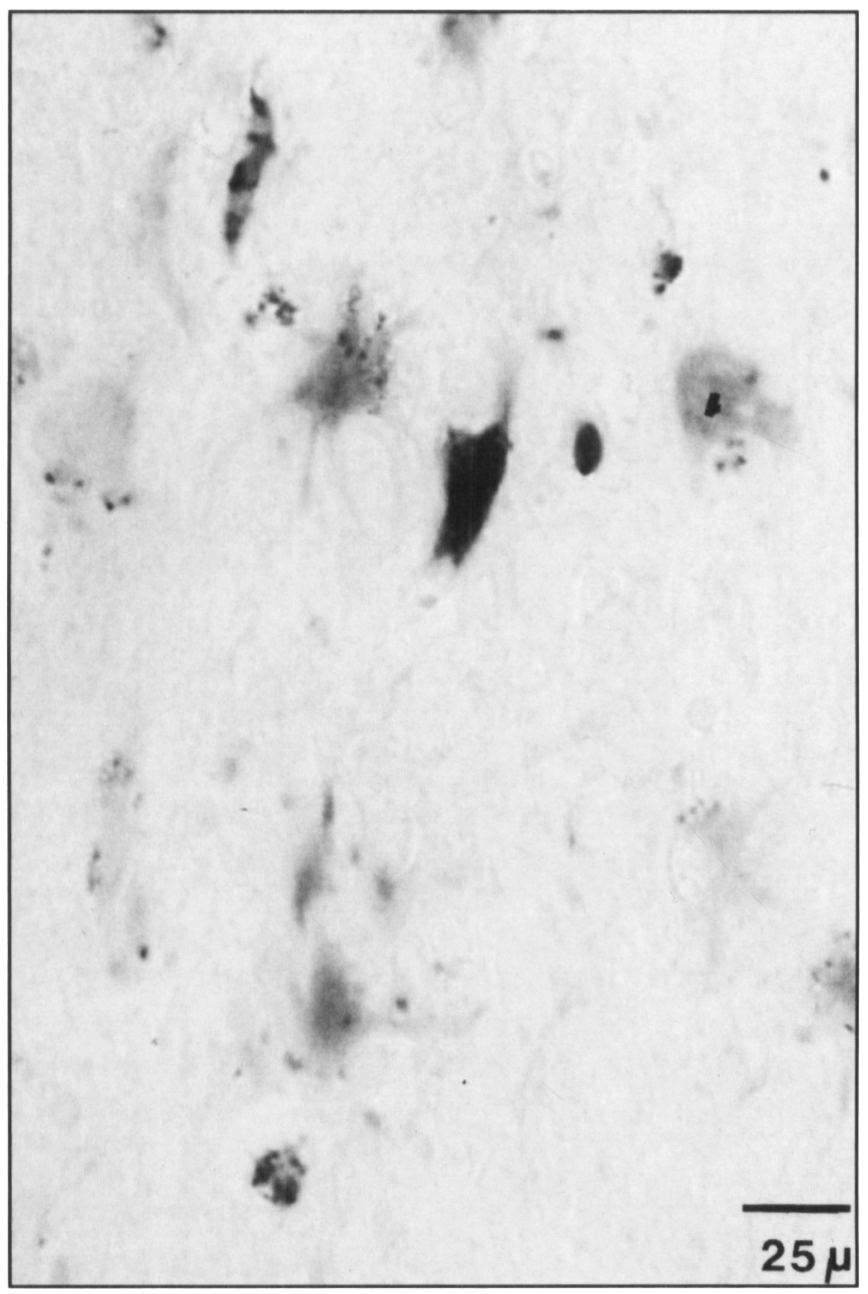

Figure 8: Putamen from patient \#2: Perls' positive iron deposition (blue) occurs independently of reactive astrocytes (brown) (GFAP/Perls', 400X).

the 8 clinically diagnosed patients who demonstrated supportive PET scan disturbances. Assuming that pathological review confirms a similar diagnosis in both groups, as more cases studied by MRI reach post mortem assessment, it will be important to evaluate whether there are clinical, pathological or etiological differences between MSA patients with and without this MRI picture.

There has been much speculation on the nature of these MRI abnormalities in MSA (diagnosed clinically). MRI has the advantage over $\mathrm{CT}$ of allowing characterization of brain tissue at the molecular level. Electromagnetic compounds such as iron will decrease signal intensity because of the local inhomogeneity they create in the magnetic field. Iron is one of the key trace metals involved in brain function and indeed there is more iron per mg of tissue in the basal ganglia and substantia nigra (SN) than in the liver (which is the major storage site of iron). ${ }^{20,21}$ Increased iron levels have been found in the $\mathrm{SN}$ and striatum in $\mathrm{MSA}^{22}$ and, therefore, most speculation has suggested that increased iron may be responsible for the MRI abnormalities. ${ }^{23}$ There are a variety of other compounds which could potentially account for this signal loss such as copper, manganese, cobalt, melanin and lipofuscin. ${ }^{10}$ Our results, however, provide good

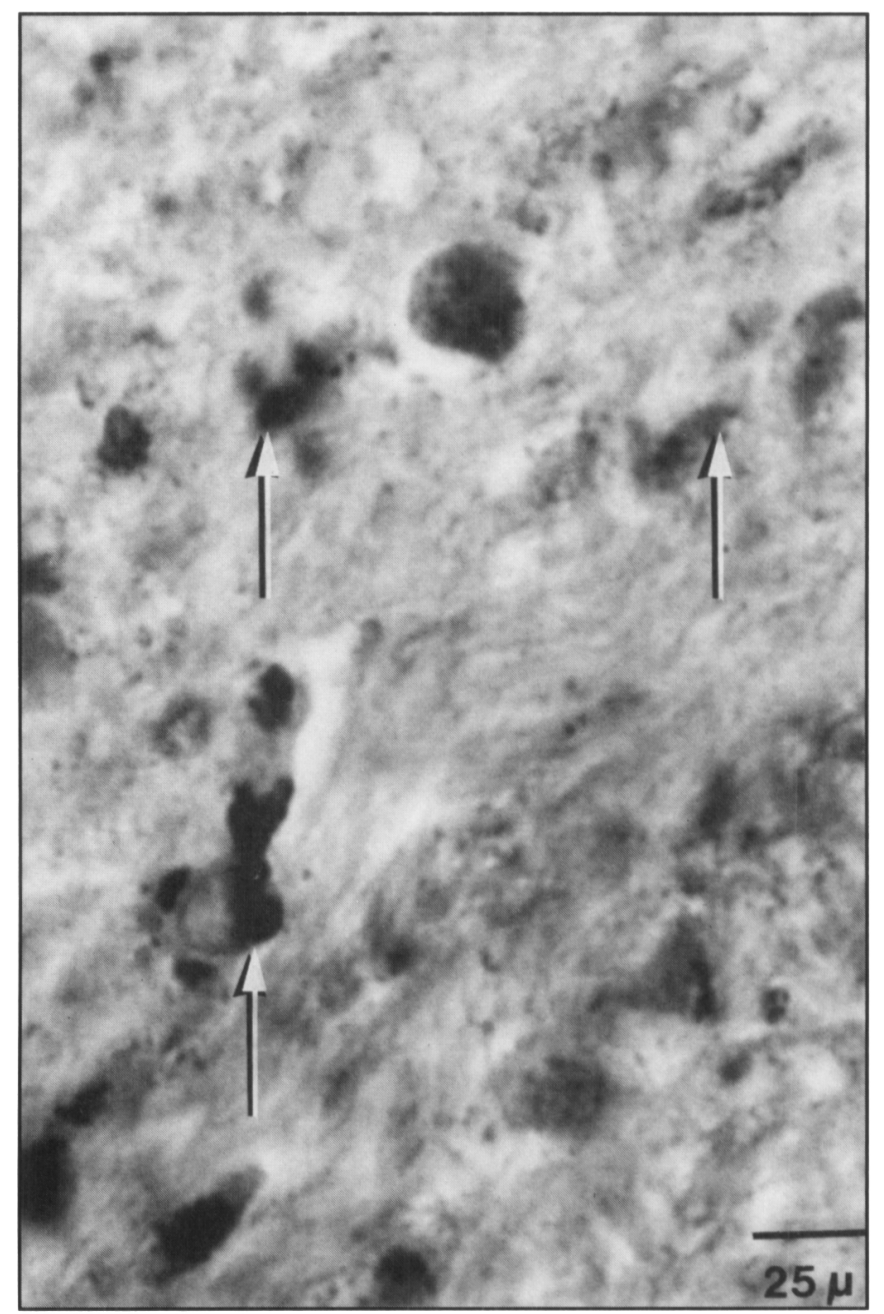

Figure 9: Reactive microglial cells containing Perls' positive iron deposition (arrows) in patient \#2 (Ferritin/Perls', 400X).

support for the role of iron in causing the MRI changes. All three cases demonstrated a significant increase in iron deposition (Perls' stain) in the putamen and this correlated well anatomically with the MRI signal loss. Others have shown the same correlation of increased iron and MRI signal loss in other disease entities such as Hallervorden Spatz disease. ${ }^{24}$ However, recent studies quantitating iron and ferritin have found no correlation between these levels and the extent of $\mathrm{T} 2$ shortening in the putamen and globus pallidus of normals and patients with Parkinson's disease and Huntington's disease. ${ }^{25.26}$

In our experience, the pattern of iron deposition in SND correlates with the extent of histological damage, the gross pigmentation of the putamen and the altered MRI signal intensity. Although the regional distribution of iron within the normal brain has been defined and the putamen is well established as an iron rich region there is little understanding of its cellular and subcellular distribution. ${ }^{10.20}$ In these 3 SND cases double labelling utilizing Perls' reaction with antisera to GFAP and ferritin showed most of the iron deposition in a network of microglial processes as well as scattered in neuropil and there was little or no iron within reactive astrocytes or residual large neurons. 


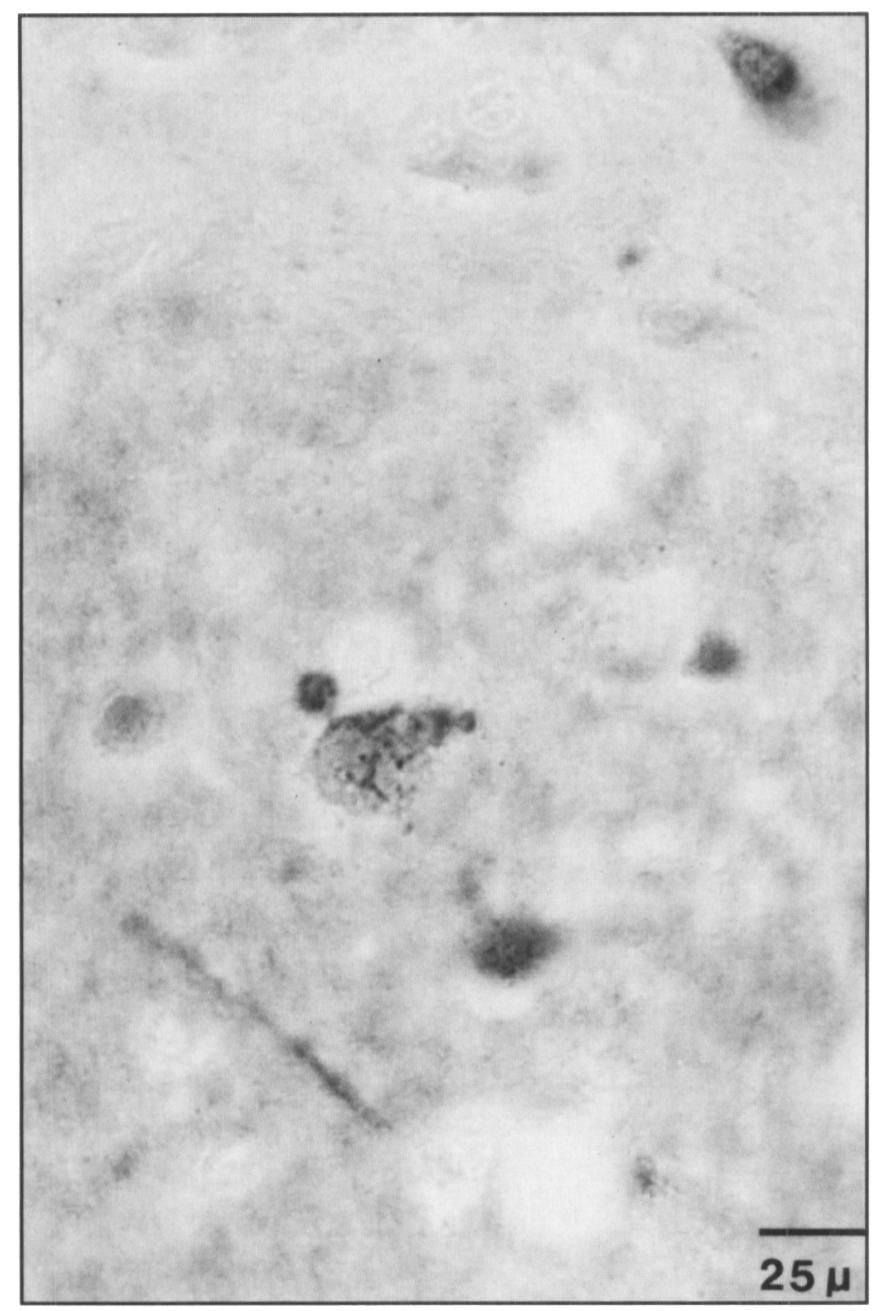

Figure 10: Residual neuron in putamen, containing cytoplasmic Perls' positive iron (NSE/Perls', 400X). From patient \#2.

Proliferation or activation of microglial cells is a wellestablished phenomenon in neurodegenerative disorders, and this has been described in Parkinson's disease, Alzheimer's disease, and ALS. ${ }^{27}$ The use of ferritin as a marker for microglial cells has previously been established under both normal and pathologic conditions. ${ }^{28}$ Thus, the extensive ferritin immunoreactivity observed in our cases may simply identify the increased microglial population and does not necessarily indicate an abnormality in iron metabolism. The microglial staining also closely parallels the amount of neuronal loss and astrocytic gliosis. Taken together these results suggest that most of the iron deposition observed histologically represents redistribution of normal intrinsic putaminal iron consequent to neuronal death. Subsequently uptake and processing by reactive microglial cells takes place allowing for the predominant localization within their cellular processes.

However, the presence of the very occasional but definite cytoplasmic Perls' staining in residual large putaminal neurons is of interest and suggests the possibility of increased intraneuronal ferric iron prior to cell death. Thus, a primary alteration in putaminal iron content, metabolism or transport cannot be completely excluded.
Independent of whether the changes in local iron metabolism are a primary or a secondary process, they may contribute to ongoing neuronal damage and death. It has been hypothesized that increased iron may promote free radical formation by facilitating oxidative reactions. ${ }^{29}$ Studies of protective therapy are being actively pursued in neurodegenerative diseases, many directed at preventing free radical formation or protecting against the consequences of their excessive formation..$^{30}$ Manipulation of brain iron, akin to aluminum chelation in Alzheimer's disease, ${ }^{31}$ may eventually prove beneficial in parkinsonism. Our results suggest that in SND, MRI has the potential to serve as an important tool in the ongoing analysis of such interventions by monitoring signal alterations in the basal ganglia.

\section{REFERENCES}

1. Hughes AJ, Daniel SE, Kilford L, Lees AJ. Accuracy of clinical diagnosis of idiopathic Parkinson's disease. J Neurol Neurosurg Psychiatry 1992; 55: 181-184.

2. Drayer BP, Olanow W, Burger P, et al. Parkinson plus syndrome: diagnosis using high field MR imaging of brain iron. Radiology 1986; 159: 493-498.

3. Stern MB, Braffman BH, Skolnick BE, Hurtig HI, Grossman RI. Magnetic resonance imaging in Parkinson's disease and parkinsonian syndromes. Neurology 1989; 39: 1524-1526.

4. Parati EA, Fetoni V, Geminiani GC, et al. Response to L-Dopa in Multiple System Atrophy. Clin Neuropharmacol 1993; 16: 139 . 144.

5. Pasatakia B, Polinsky R, De Chiro G, et al. Multiple system atrophy (Shy-Drager syndrome): MR imaging. Radiology 1990; 174: 609-696.

6. Guttman M, Lang AE, Garnett ES, et al. Regional cerebral glucose metabolism in SLE chorea: further evidence that striatal hypometabolism is not the correlate of chorea. Mov Disord 1987; 2: 201-210.

7. Garnett ES, Lang AE, Chirakal R, Firnau G, Nahmias C. A rostrocaudal gradient for aromatic acid decarboxylase in the human striatum. Can J Neurol Sci 1987; 14: 444-447.

8. Papp MI, Kahn JE, Lantos PL. Glial cytoplasmic inclusions in the CNS of patients with multiple system atrophy (striatonigral degeneration, olivopontocerebellar atrophy and Shy-Drager Syndrome). J Neurol Sci 1989; 94: 79-100.

9. Lantos PL, Papp MI. Cellular pathology of multiple system atrophy: a review. J Neurol Neurosurg Psychiatry 1994; 57: 129-133.

10. Hock A, Demmel U, Schicha K. Trace element concentration in human brain. Brain 1975; 98: 49-64.

11. Morris CM, Candy JM, Bloxham CA, Edwardson JA. Immunocytochemical localization of transferrin in the human brain. Acta Anatomica 1992; 143: 14-18.

12. Hughes AJ, Colosimo C, Kleedorfer B, Daniel SE. Lees AJ. The dopaminergic response in multiple system atrophy. J Neurol Neurosurg Psychiatry 1992; 55: 1009-1013.

13. Riley DE, Lang AE, Lewis A, et al. Cortical-basal ganglionic degeneration. Neurology 1990; 40: 1203-1212.

14. Chen R, Ashby P, Lang AE. Stimulus-sensitive myoclonus in akinetic-rigid syndromes. Brain 1992; 115: 1875-1888.

15. Rodrigues ME, Artieda J, Zubieta JL, Obeso JA. Reflex myoclonus in olivopontocerebellar atrophy. J Neurol Neurosurg Psychiatry 1994: 57: 316-319.

16. Brooks DJ, lbanez V, Sawle GV, et al. Differing patterns of striatal ${ }^{18} \mathrm{~F}$-Dopa Uptake in Parkinson's Disease, Multiple System Atrophy, and Progressive Supranuclear Palsy. Ann Neurol 1990; 28: 547-555.

17. Burn DJ, Sawle GV, Brooks DJ. Differential diagnosis of Parkinson's disease, multiple system atrophy and SteeleRichardson-Olszewski syndrome: discriminant analysis of striatal ${ }^{18}$ F-dopa PET data. J Neurol Neurosurg Psychiatry 1994: 57: 278-284.

18. Eidelberg D, Takikawa S, Moeller JR, et al. Striatal hypometabolism distinguishes striatonigral degeneration from Parkinson's disease. Ann Neurol 1993; 33: 518-527. 
19. O`Brien C, Sung JH, McGeachie RE, Lee MC. Striatonigral degeneration: clinical, MRI and pathological correlation. Neurology 1990; 40: 710-711.

20. Hallgren B, Sourander $P$. The effect of age on the nonhaem iron in human brain. J Neurochem 1958; 3: 41-51.

21. Perl DP, Good BS. Comparative techniques for determining cellular iron distribution in brain tissues. Ann Neurol 1992; 32: S76-S81.

22. Kato S, Meshitsuka S, Ohama E, et al. Increased iron content in the putamen of patients with striatonigral degeneration. Acta Neuropathologica 1992; 84: 328-330.

23. Drayer BP. Magnetic resonance imaging and brain iron: Implications in the diagnosis and pathochemistry of movement disorders and dementia. BNI Quarterly 1987; 3: 15-30.

24. Schafert DA, Johnsen SD, Johnson PC, Drayer BP. Magnetic resonance imaging in pathologically proven Hallervorden-Spatz disease. Neurology 1989; 39: 440-442.

25. Chen JC, Hardy PA, Kucharczyk W, et al. MR of human postmortem brain tissue: correlative study between $\mathrm{T} 2$ and assays of iron and ferritin in Parkinson and Huntington disease. Am J Neuroradiol 1993; 14: 275-281.
26. Chen JC, Hardy PA, Clauberg M, et al. T2 values in the human brain: comparison with quantitative assays of iron and ferritin. Radiology 1989; 173: 521-526.

27. McGeer PL, Akiyama H, Itagaki S, McGeer EG. Immune system response in Alzheimer's disease. Can J Neurol Sci 1989; 16: 516-527.

28. Kaneko Y, Kitamoto T, Tateishi J. Ferritin immunohistochemistry as a marker for microglia. Acta Neuropathol (Berlin) 1989; 79: 129.136.

29. Halliwell B, Gutteridge JMC. Iron and free radical reactions: two aspects of antioxidant protection. Trends Biochem Sci 1986; 11: 1372-1375.

30. The Parkinson Study Group. Effect of deprenyl on the progression of disability in early Parkinson's disease. N Engl J Med 1989; 321: 1364-1371.

31. McLachlan DRC, Dalton AJ, Kruck TPA, Bell MY, Smith WL. Intramuscular desferrioxamine in patients with Alzheimer's disease. Lancet 1991; 337: 1304-1308. 